\title{
Psychological vulnerability to stress in carriers of congenital adrenal hyperplasia due to 21-hydroxylase deficiency
}

\author{
Eleni-Magdalini Kyritsi, ${ }^{1 *}$ Georgia Koltsida, ${ }^{1 *}$ Ioanna Farakla, ${ }^{1}$ Aikaterini Papanikolaou, ${ }^{2}$ \\ Elena Critselis, ${ }^{1}$ Emilia Mantzou, ${ }^{1}$ Emmanouil Zoumakis, ${ }^{1}$ Gerasimos Kolaitis, ${ }^{2}$ \\ George P. Chrousos, ${ }^{1,3}$ Evangelia Charmandari ${ }^{1,3}$
}

${ }^{1}$ Division of Endocrinology, Metabolism and Diabetes, First Department of Pediatrics, National and Kapodistrian University of Athens Medical School, 'Aghia Sophia' Children's Hospital, Athens, Greece, ${ }^{2}$ Department of Child Psychiatry, National and Kapodistrian University of Athens Medical School, 'Aghia Sophia' Children's Hospital, Athens, Greece, ${ }^{3}$ Division of Endocrinology and Metabolism, Clinical Research Center, Biomedical Research Foundation of the Academy of Athens, Athens, Greece

*The authors contributed equally to this study.

\begin{abstract}
OBJECTIVE: Carriers of congenital adrenal hyperplasia (CAH) due to 21-hydroxylase deficiency (21-OHD) demonstrate increased secretion of cortisol precursors following ACTH stimulation, suggestive of impaired cortisol production and compensatory increases in hypothalamic corticotropin-releasing hormone (CRH) secretion. Both cortisol and CRH have behavioral effects, and hypothalamic CRH hypersecretion has been associated with chronic states of anxiety and depression. We performed an endocrinologic and psychological evaluation in carriers of 21-OHD and matched control subjects. DESIGN: We recruited 29 parents of children with classic CAH (14 males, 15 females; age (mean \pm SD): $41.8 \pm 5.7$ yr), and hence 21-OHD carriers, and 13 normal subjects (5 males, 8 females; age: $43.8 \pm 6.1$ yr). All subjects underwent a formal ovine (o) CRH stimulation test with measurement of ACTH, cortisol, 17-hydroxyprogesterone (17-OHP) and androstenedione concentrations, which was preceded by determination of 24-hour urinary free cortisol (UFC) excretion. Psychometric assessment was performed by administering the State-Anxiety (STAI 1) and Trait-Anxiety (STAI 2) Inventory, Beck Depression Inventory, Symptom Checklist-90R and Temperament and Character Inventory. RESULTS: Carriers of 21-OHD had significantly higher 17-OHP concentrations following oCRH stimulation and higher STAI $1(47.6 \pm 5.6$ vs. $43.3 \pm 5.4, P=0.023)$ scores than control subjects. Mean 24-hour UFC concentrations were positively correlated with paranoid
\end{abstract}

Address for correspondence:

Evangelia Charmandari, MD, MSc, PhD, MRCP (UK), CCST (UK), Division of Endocrinology, Metabolism and Diabetes, First

Department of Pediatrics, National and Kapodistrian University of Athens Medical School, 'Aghia Sophia' Children's Hospital, Thivon and Papadiamantopoulou Street, Athens, 11527, Greece; Tel.: +30-213-2013 384 and +30-6947904369; Fax: 213-2013 384,

E-mail: evangelia.charmandari@googlemail.com

Received: 05-11-2016, Accepted: 28-02-2017 
ideation $(\mathrm{r}=0.435 ; P=0.023)$ and psychoticism $(\mathrm{r}=0.454 ; P=0.017)$. Stepwise multiple linear regression analysis revealed that the single independent predictor of STAI 1 was peak stimulated 17-OHP concentrations $\left(\beta: 0.055, \mathrm{SE}: 0.023, \mathrm{R}^{2}: 0.290, P=0.031\right)$. CONCLUSIONS: Carriers of 21-OHD may be predisposed to the development of anxiety disorders.

Key words: Anxiety, Carriers, Congenital adrenal hyperplasia, Depression, 21-hydroxylase deficiency

\section{INTRODUCTION}

Congenital adrenal hyperplasia $(\mathrm{CAH})$ due to 21-hydroxylase deficiency (21-OHD) is an autosomal recessive disorder characterized by decreased glucocorticoid and often mineralocorticoid secretion from the adrenal cortex owing to deletions or mutations of the cytochrome P450 21-hydroxylase (CYP21A2) gene. The impaired glucocorticoid feedback inhibition at the hypothalamic and anterior pituitary levels leads, respectively, to compensatory hypersecretion of corticotropin-releasing hormone $(\mathrm{CRH})$ and argininevasopressin (AVP) in the hypophyseal portal system, and increased secretion of adrenocorticotropic hormone $(\mathrm{ACTH})$ in the systemic circulation. The latter results in adrenocortical hyperplasia and increased production of adrenal androgens and steroid precursors prior to the enzymatic defect. The clinical spectrum of the condition is broad, ranging from severe to mild forms depending on the degree of 21-hydroxylase activity. Accordingly, three main clinical phenotypes have been described: classic salt-wasting, classic simplevirilizing and non-classic or late-onset $\mathrm{CAH} .{ }^{1-4}$ The overall incidence of classic $\mathrm{CAH}$ is approximately one in 15,000 live births; however, it varies considerably among ethnic groups. ${ }^{1}$ Thus, the carrier frequency of classic CAH ranges from 1:20 to 1:60 individuals. The non-classic form occurs in approximately $0.2 \%$ of the general Caucasian population but is more frequent $(1-2 \%)$ in certain populations, such as Jews of Eastern European origin. ${ }^{1,2,5}$

Heterozygocity of 21-OHD has been associated with mild hormonal abnormalities, which reflect an up-regulation of the hypothalamic-pituitary axis. Compared with normal subjects, carriers of 21-OHD have lower 24-hour urinary free cortisol (UFC) excretion and higher $08: 00 \mathrm{~h}$ total and free testosterone concentrations. Furthermore, they demonstrate increased 17-hydroxyprogesterone (17-OHP) concentrations following ACTH stimulation, and higher mean and peak ACTH and 17-OHP concentrations following ovine (o) CRH stimulation. ${ }^{1,6,7}$ These findings are likely to reflect chronic mild hypothalamic $\mathrm{CRH}$ and/or AVP hypersecretion secondary to mild impairment in cortisol biosynthesis, which is compensated for by the increased activity of the hypothalamic-pituitary axis. ${ }^{7}$

The hypothalamic-pituitary-adrenal (HPA) axis represents a major component of the stress system and plays an important role in maintaining basal and stress-related homeostasis. ${ }^{8-10} \mathrm{CRH}$, a 41-amino-acid neuropeptide secreted by the parvocellular neurons of the paraventricular nucleus of the hypothalamus, is the primary regulator of HPA axis activity and the central coordinator of the endocrine, autonomic, immune and behavioral responses to stress. ${ }^{911-13}$ Both hypothalamic and extra-hypothalamic CRH neurons may play a substantial role in the development of and/or vulnerability to stress-related disorders. ${ }^{8,9,14}$ Accumulating evidence suggests that increased HPA axis activity - and, hence, inferred CRH hypersecretion-is associated with the development of several affective and anxiety disorders, including generalized anxiety disorder (GAD), major depressive disorder, panic disorder (PD), anorexia, obsessive-compulsive disorder (OCD) and alcoholism..$^{9,13-15}$ The development and severity of these conditions primarily depend on the genetic vulnerability of the individual, exposure to adverse environmental factors and the timing of the stressful event(s), given that prenatal life, infancy, childhood and adolescence are critical periods characterized by increased vulnerability to stressors. Therefore, genetic predisposition coupled with stress during critical phases of development may result in a phenotype that is neurobiologically vulnerable to stress and may lower an individual's threshold for developing depressive and anxiety disorders upon subsequent exposure to stress.

The aim of the present study was to determine the endocrinologic and psychological profile of car- 
riers of 21-OHD and matched control subjects and to investigate the association between their adrenocortical responses to oCRH stimulation and various psychometric parameters determined at psychological evaluation.

\section{SUBJECTS AND METHODS}

\section{Subjects}

Twenty-nine consecutive parents of children with $\mathrm{CAH}$ due to 21-OHD and, hence, carriers of 21-OHD [14 males and 15 females; age \{mean \pm standard deviation (SD) $\}: 41.8 \pm 5.7 \mathrm{yr}]$ and 13 normal subjects (5 males and 8 females; age: $43.8 \pm 6.1 \mathrm{yr}$ ) were studied prospectively. The carrier state of 21-OHD was confirmed by genotype. All normal subjects were parents of children with a chronic endocrine disorder, such as Turner syndrome $(\mathrm{n}=1)$ or hypothyroidism $(\mathrm{n}=12)$ and were of similar ethnicity and socioeconomic status to the carriers of 21-OHD. No subject had a family history of mental illness or evidence of hepatic or renal disease and none was receiving psychotropic medications, or medications known to influence HPA axis activity or corticosteroid-binding globulin (CBG) concentrations, or to induce hepatic enzymes. The clinical characteristics of all subjects are summarized in Table 1. The study was approved by the local Committee on the Ethics of Human Research and written informed consent was obtained in all cases.

\section{Methods}

All subjects were admitted to the Endocrine Unit and standard anthropometric, hematologic and biochemical measurements were obtained. All subjects fasted for 12 hours before blood samples were collected.

On the first and second days of the study, saliva specimens were obtained at 08:00h, 12:00h, 16:00h, 20:00h and 24:00h for measurement of cortisol concentrations, and 24-hour urine specimens were collected for determination of UFC excretion. On the third day, an indwelling venous catheter for blood sampling was inserted $1 \mathrm{~h}$ before sampling and all subjects rested in a supine position before blood samples were collected to allow for a period of adaptation. Subsequently, oCRH was given intravenously $(1 \mu \mathrm{g} / \mathrm{kg})$ at $08: 00 \mathrm{~h}$ and blood samples for ACTH, cortisol, 17-OHP and androstenedione concentrations were collected at -5 ,
$0,15,30,45,60,90$ and 120 min after stimulation. An additional blood sample for measurement of Glucose, Insulin, TSH, T3, Free T4, Prolactin, Testosterone, Estradiol, Dehydroepiandrosterone sulfate (DHEA-S) and $\mathrm{CBG}$ concentrations was drawn at 08:00h, prior to the administration of oCRH. Samples were centrifuged and separated immediately after collection and were stored at $-80^{\circ} \mathrm{C}$ until assayed.

\section{Psychometric assessment}

The following instruments were administered to all subjects at baseline:

State-Anxiety Inventory (STAI): The STAI consists of 20 items to assess state anxiety (STAI 1) and another 20 items to assess trait anxiety (STAI 2). State anxiety is defined as an unpleasant emotional arousal in face of threatening demands or dangers. A cognitive appraisal of threat is a prerequisite for the experience of this emotion. Trait anxiety, on the other hand, reflects the existence of stable individual differences in the tendency to respond with state anxiety in the anticipation of threatening situations. ${ }^{16}$

Beck Depression Inventory (BDI): The BDI is a commonly used self-report rating instrument for quantifying levels of depression; ${ }^{17}$ in the first part of the test, psychological symptoms are assessed, whereas the second part assesses physical symptoms. The BDI includes 21 items in which patients are asked to answer on a four-point scale ranging from 0 (symptom not present) to 3 (symptom very intense). The BDI demonstrates high internal consistency, with Cronbach alpha values of .86 and .81 for psychiatric and non-psychiatric populations, respectively. ${ }^{17}$

Symptom Checklist-90R (SCL90-R): This is a 90 -item multidimensional rating scale designed to measure current symptomatology and distress. Subjects indicated the amount of disturbance a particular item produced during the past week on a Likert scale ranging from 0 (not at all) to 4 (extremely). An average of all items is used as a global measure of distress. In addition, there are 9 symptom clusters (comprised of 10 items each): somatization, obsessive-compulsive, interpersonal sensitivity, depression, anxiety, hostility, phobic anxiety, paranoid ideation and psychoticism. Scores for each are derived from the average of each symptom cluster's items. ${ }^{18}$ 
Table 1. Clinical characteristics and endocrinologic parameters in carriers of 21-hydroxylase deficiency (21-OHD) and control subjects

\begin{tabular}{|c|c|c|c|}
\hline & Carriers of 21-OHD $(n=29)$ & Control subjects $(n=13)$ & $P$-value* \\
\hline Age (years) & $41.8 \pm 5.8$ & $43.8 \pm 6.1$ & 0.332 \\
\hline Gender & & & 0.7399 \\
\hline Male & $14(48.3 \%)$ & $5(38.5 \%)$ & \\
\hline Female & $15(51.7 \%)$ & $8(61.5 \%)$ & \\
\hline Body mass index (BMI, $\left.\mathrm{kg} / \mathrm{m}^{2}\right)$ & $26.9 \pm 5.4$ & $24.1 \pm 3.6$ & 0.141 \\
\hline \multicolumn{4}{|l|}{$\mathrm{ACTH}(\mathrm{pmol} / \mathrm{L})$} \\
\hline-15 minutes & $3.9 \pm 3.1$ & $2.2 \pm 1.1$ & 0.094 \\
\hline 0 minutes & $2.8 \pm 1.5$ & $2.0 \pm 1.7$ & 0.089 \\
\hline 15 minutes & $7.9 \pm 7.5$ & $8.3 \pm 6.6$ & 0.918 \\
\hline 30 minutes & $7.8 \pm 4.8$ & $8.2 \pm 4.5$ & 0.691 \\
\hline 45 minutes & $6.4 \pm 2.7$ & $6.8 \pm 3.3$ & 0.735 \\
\hline 60 minutes & $4.7 \pm 1.8$ & $5.4 \pm 2.9$ & 0.627 \\
\hline 90 minutes & $3.1 \pm 1.4$ & $3.1 \pm 2.1$ & 0.508 \\
\hline 120 minutes & $2.4 \pm 1.3$ & $2.4 \pm 1.4$ & 0.566 \\
\hline \multicolumn{4}{|l|}{ 17-OHP (nmol/L) } \\
\hline-15 minutes & $8.2 \pm 5.2$ & $4.2 \pm 2.4$ & 0.001 \\
\hline 0 minutes & $7.1 \pm 3.8$ & $4.2 \pm 2.4$ & 0.003 \\
\hline 15 minutes & $9.1 \pm 9.4$ & $4.8 \pm 2.7$ & 0.004 \\
\hline 30 minutes & $11.2 \pm 9.4$ & $5.5 \pm 2.7$ & 0.001 \\
\hline 45 minutes & $12.1 \pm 9.7$ & $5.8 \pm 3.3$ & $<0.0001$ \\
\hline 60 minutes & $10.0 \pm 5.2$ & $5.5 \pm 3.0$ & 0.001 \\
\hline 90 minutes & $7.9 \pm 4.2$ & $4.8 \pm 2.7$ & 0.010 \\
\hline 120 minutes & $7.3 \pm 3.9$ & $4.5 \pm 2.7$ & 0.006 \\
\hline \multicolumn{4}{|l|}{ Cortisol (nmol/L) } \\
\hline-15 minutes & $372.5 \pm 168.3$ & $314.5 \pm 38.6$ & 0.588 \\
\hline 0 minutes & $309.0 \pm 143.5$ & $281.4 \pm 44.1$ & 0.955 \\
\hline 15 minutes & $438.7 \pm 126.9$ & $372.5 \pm 91.0$ & 0.081 \\
\hline 30 minutes & $516.0 \pm 121.4$ & $488.3 \pm 110.4$ & 0.586 \\
\hline 45 minutes & $546.3 \pm 110.4$ & $524.2 \pm 113.1$ & 0.607 \\
\hline 60 minutes & $502.1 \pm 104.8$ & $535.2 \pm 121.4$ & 0.489 \\
\hline 90 minutes & $386.3 \pm 74.5$ & $438.7 \pm 110.4$ & 0.134 \\
\hline 120 minutes & $306.2 \pm 93.8$ & $331.1 \pm 104.8$ & 0.988 \\
\hline
\end{tabular}

Continuous variables presented as mean \pm standard deviation $(\mathrm{SD}) ;$ *Mann-Whitney $\mathrm{p}$-value; $\uparrow$ Chi-squared p-value. Reference ranges: ACTH: 2.0-11.5 pmol/L; 17-OHP: Females Follicular phase: 0.3-3.3 nmol/L, Luteal phase: 2.9-13.0 nmol/L, Males: 0.8-3.6 nmol/L; Cortisol: 138-552 nmol/L; Androstenedione: Females: 1.9-8.7 nmol/L, Males: 2.1-10.8 nmol/L; Testosterone: Females: 0.347-4.16

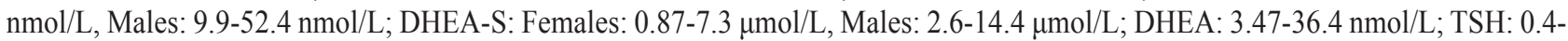
$4 \mathrm{mIU} / \mathrm{L}$; FT3: 2.46-7.8 pmol/L; FT4: 9-20.5 pmol/L; PRL: Adult females: 0.08-1.09 nmol/L, Adult males: 0.11-0.74 nmol/L; CBG: Females: 687.2-2645.7 nmol/L, Males: $378.0-945.0 \mathrm{nmol} / \mathrm{L}$; Glucose: 3.9-6.7 mmol/L; Insulin: 28.7-193.7 pmol/L; Cortisol, Urine: 13.8-179.4 nmol/day; Cortisol, Salivary: Morning hours: 8-10 a.m: $<19.1 \mathrm{nmol} / \mathrm{L}$, Afternoon hours: 2:30-3:30 p.m: $<11.9 \mathrm{nmol} / \mathrm{L}$, Midnight: $8.33 \pm 3.62 \mathrm{nmol} / \mathrm{L}$. 
Table 1. (continued) Clinical characteristics and endocrinologic parameters in carriers of 21-hydroxylase deficiency (21-OHD) and control subjects

\begin{tabular}{|c|c|c|c|}
\hline & Carriers of 21-OHD $(n=29)$ & Control subjects $(n=13)$ & $P$-value* \\
\hline \multicolumn{4}{|l|}{ Androstenedione (nmol/L) } \\
\hline-15 minutes & $8.4 \pm 4.5$ & $5.2 \pm 2.5$ & 0.036 \\
\hline 0 minutes & $7.0 \pm 3.1$ & $5.2 \pm 2.8$ & 0.052 \\
\hline 15 minutes & $8.0 \pm 3.5$ & $6.3 \pm 2.4$ & 0.168 \\
\hline 30 minutes & $9.4 \pm 3.8$ & $7.7 \pm 3.1$ & 0.142 \\
\hline 45 minutes & $10.5 \pm 3.5$ & $8.0 \pm 3.5$ & 0.093 \\
\hline 60 minutes & $9.8 \pm 3.1$ & $8.4 \pm 4.2$ & 0.208 \\
\hline 90 minutes & $7.7 \pm 2.4$ & $7.0 \pm 4.2$ & 0.323 \\
\hline 120 minutes & $6.3 \pm 2.8$ & $5.6 \pm 2.8$ & 0.435 \\
\hline Testosterone (nmol/L) & $6.9 \pm 6.2$ & $7.6 \pm 10.4$ & 0.901 \\
\hline DHEA-S $(\mu \mathrm{mol} / \mathrm{L})$ & $4.0 \pm 2.5$ & $3.3 \pm 2.0$ & 0.750 \\
\hline DHEA (nmol/L) & $25.7 \pm 13.2$ & $20.8 \pm 11.8$ & 0.353 \\
\hline TSH (mIU/L) & $1.4 \pm 0.8$ & $1.4 \pm 0.6$ & 0.836 \\
\hline Free T3 (pmol/L) & $5.2 \pm 0.8$ & $4.9 \pm 0.5$ & 0.226 \\
\hline Free T4 (pmol/L) & $14.3 \pm 2.0$ & $14.4 \pm 1.2$ & 0.901 \\
\hline Prolactin (nmol/L) & $0.31 \pm 0.2$ & $0.30 \pm 0.1$ & 0.879 \\
\hline $\mathrm{CBG}(\mathrm{nmol} / \mathrm{L})$ & $891.6 \pm 240.7$ & $891.6 \pm 204.4$ & 0.913 \\
\hline Glucose (mmol/L) & $5.1 \pm 0.7$ & $5.1 \pm 0.1$ & 0.977 \\
\hline Insulin (pmol/L) & $43.1 \pm 68.2$ & $33 \pm 14.4$ & 0.146 \\
\hline Cortisol, Urine (nmol/day) & $101.9 \pm 64.1$ & $98.3 \pm 75.7$ & 0.874 \\
\hline \multicolumn{4}{|l|}{ Cortisol, Salivary (nmol/L) } \\
\hline $8: 00 \mathrm{~h}$ & $19.3 \pm 15.1$ & $11.0 \pm 5.5$ & 0.130 \\
\hline $12: 00 \mathrm{~h}$ & $8.3 \pm 8.3$ & $5.5 \pm 0.3$ & 0.652 \\
\hline $16: 00 \mathrm{~h}$ & $11.0 \pm 16.6$ & $2.8 \pm 0.3$ & 0.067 \\
\hline $20: 00 \mathrm{~h}$ & $5.5 \pm 5.5$ & $2.8 \pm 0.3$ & 0.587 \\
\hline $24: 00 \mathrm{~h}$ & $5.5 \pm 2.8$ & $5.5 \pm 8.3$ & 0.691 \\
\hline
\end{tabular}

Continuous variables presented as mean \pm standard deviation (SD); *Mann-Whitney p-value; $\uparrow$ Chi-squared p-value. Reference ranges: ACTH: 2.0-11.5 pmol/L; 17-OHP: Females Follicular phase: 0.3-3.3 nmol/L, Luteal phase: $2.9-13 \mathrm{nmol} / \mathrm{L}$, Males: 0.8-3.6 nmol/L; Cortisol: 138-552 nmol/L; Androstenedione: Females: 1.9-8.7 nmol/L, Males: 2.1-10.8 nmol/L; Testosterone: Females: 0.347-4.16 nmol/L, Males: 9.9-52.4 nmol/L; DHEA-S: Females: 0.87-7.3 $\mu \mathrm{mol} / \mathrm{L}$, Males: 2.6-14.4 $\mu \mathrm{mol} / \mathrm{L}$; DHEA: 3.47-36.4 nmol/L; TSH: 0.44 mIU/L; FT3: 2.46-7.8 pmol/L; FT4: 9-20.5 pmol/L; PRL: Adult females: 0.08-1.09 nmol/L, Adult males: 0.11-0.74 nmol/L; CBG: Females: 687.2-2645.7 nmol/L, Males: 378.0-945.0 nmol/L; Glucose: 3.9-6.7 mmol/L; Insulin: 28.7-193.7 pmol/L; Cortisol, Urine: 13.8-179.4 nmol/day; Cortisol, Salivary: Morning hours: 8-10 a.m: <19.1 nmol/L, Afternoon hours: 2:30-3:30 p.m: <11.9 nmol/L, Midnight: $8.33 \pm 3.62 \mathrm{nmol} / \mathrm{L}$.

Temperament and Character Inventory (TCI): This is a 240 -item questionnaire to assess personality style. The TCI describes normal personality by 4 putatively inborn temperamental dimensions (novelty seeking, harm avoidance, reward dependence and persistence) and 3 character dimensions (self-directedness, cooperativeness and self-transcendence). ${ }^{19}$

All the questionnaires we used for the purpose of this study were in Greek and have been standardized and validated accordingly. ${ }^{20-23}$ 
Assays

Cortisol: Serum cortisol concentrations were determined using a two-site Chemiluminescent Immunoassay CLIA (Immulite 2000, SIEMENS Healthcare Diagnostics Products Ltd, Gwynedd, UK) with a sensitivity of $5.5 \mathrm{nmol} / \mathrm{L}$. The intra-assay coefficients of variation (CVs) were $6.1 \%, 5.3 \%$ and $7.4 \%$ at 91.0 , 496.6 and $855.3 \mathrm{nmol} / \mathrm{L}$, respectively. The inter-assay CVs were $8.2 \%, 7.2 \%$ and $9.4 \%$ at $91.0,496.6$ and $855.3 \mathrm{nmol} / \mathrm{L}$, respectively.

Urinary Free Cortisol (UFC): UFC was determined by a competitive direct chemiluminescent assay (ADVIA Centaur System, Siemens, Erlangen, Germany) with a sensitivity of $27.6 \mathrm{nmol} / \mathrm{L}$. The intra-assay CVs were $3.69 \%, 3.09 \%$ and $2.98 \%$ at 107.1, 155.3 and $1025.0 \mathrm{nmol} / \mathrm{L}$, respectively. The inter-assay CVs were $5.45 \%, 3.83 \%, 3.07 \%$ and $1.86 \%$ at $107.1,155.3,391.0$ and $759.6 \mathrm{nmol} / \mathrm{L}$, respectively.

Salivary Cortisol: Salivary Cortisol was measured by an automated Electro-chemiluminescence Immunoassay [Analyzer Cobas e411-ROCHE DIAGNOSTICS (GmbH, Mannheim, Germany)]. The precision (within run) CV was $1.0-1.3 \%$.

ACTH: ACTH was determined by a Chemiluminescent Immunoassay CLIA (Immulite 2000, SIEMENS Healthcare Diagnostics Products Ltd., Gwynedd, UK) with intra-assay CVs of 3.1\%-9.6\% and inter-assay CVs of $5.1 \%-9.2 \%$. The sensitivity of the assay was $1.1 \mathrm{pmol} / \mathrm{L}$.

17-Hydroxyprogesterone (17-OHP): 17-OHP was measured by a Radioimmunoassay (RIA) (Diasource Immunoassays SA, Louvain-la-Neuve, Belgium) with a sensitivity of $0.06 \mathrm{nmol} / \mathrm{L}$. The intra-assay CVs were $6.2 \%, 5.6 \%$ and $5.1 \%$ at 1.30 and 7.12 and $22.88 \mathrm{nmol} / \mathrm{L}$, respectively. The inter-assay CVs were $9.2 \%$ and $5.2 \%$ at 2.33 and $5.97 \mathrm{nmol} / \mathrm{L}$, respectively.

Androstenedione: Androstenedione was measured using a Chemiluminescent Immunoassay CLIA (Immulite 2000, SIEMENS Healthcare Diagnostics Products Ltd., Gwynedd, UK) with a sensitivity of $1.05 \mathrm{nmol} / \mathrm{L}$. The intra-assay CVs were $11.3 \%, 6.7 \%$ and $4.1 \%$ at $1.85,2.61$ and $12.67 \mathrm{nmol} / \mathrm{L}$, respectively. The inter-assay CVs were $13.2 \%, 8.7 \%$ and $5.9 \%$ at $1.85,4.47$ and $27.89 \mathrm{nmol} / \mathrm{L}$, respectively.
Other Measurements: Testosterone, prolactin, DHEA-S, FSH and LH concentrations were determined by a two-site Chemiluminescent Immunoassay - CLIA (Immulite 2000, SIEMENS Healthcare Diagnostics Products Ltd., Gwynedd, UK). CBG was determined by a Radioimmunoassay (RIA) (Diasource Immunoassays SA, Louvain-la-Neuve, Belgium) with a sensitivity of $4.5 \mathrm{nmol} / \mathrm{L}$.

\section{Statistical analyses}

Continuous variables are expressed as mean $\pm \mathrm{SD}$, while categorical variables are presented as frequency and percentages. The normality of distributions of continuous variables was evaluated with the Kolmogorov-Smirnov test. The non-parametric MannWhitney U-test was applied to compare non-normally distributed continuous variables. The chi-squared test was applied to compare categorical variables between groups. The correlation between endocrine and psychometric parameters was evaluated with Pearson's correlation coefficient. Receiver operating curve (ROC) analyses were applied to calculate the area under the concentration curve (AUC) of selected endocrine parameters over time. Stepwise multiple linear regression analysis was used to investigate independent predictors of the psychometric parameters assessed. The independent variables entered into all regression models included peak or mean concentrations of ACTH, 17-OHP and androstenedione, as well as UFC and salivary cortisol. Variables retained in the model were selected according to the Wald p-value $<0.05$ criterion. The criterion of significance for all analyses was a two-tailed p-value $<0.05$. Statistical analyses were performed with SAS v. 9.2 (SAS Institute, Cary, NC, USA), and ROC analyses were performed with SPSS v. 21 (SPSS Inc.). A power analysis to determine the necessary study sample size for evaluating the primary study objective was conducted based on the CDC - Epi Info version 7.0 Software (Dean AG et. al. CDC, Atlanta, GA, USA, 2011).

\section{RESULTS}

The study population $(\mathrm{n}=42)$ consisted of 29 (69.1\%) carriers of 21-OHD and 13 (31\%) control subjects. The clinical characteristics and endocrinologic parameters of the study population are presented in 
Table 1. Carriers of 21-OHD did not differ significantly from controls with respect to age, gender or BMI. With respect to endocrinologic parameters, carriers of 21-OHD had significantly higher peak and mean concentrations of 17-OHP, as well as AUC, at all consecutive time points assessed (Table 2). However, they did not differ significantly from their healthy counterparts with respect to peak, mean or AUC concentrations of either ACTH or cortisol, including plasma, urine and salivary cortisol concentrations (Figure 1). Of note, carriers of 21-OHD had slightly higher mean ACTH values baseline (at -15 and $0 \mathrm{~min}$ ). This finding, although not statistically significant, may indicate an interesting trend.

Comparisons between carriers of 21-OHD and controls in the performance of psychometric parameters are presented in Table 3. The psychometric assessment according to the State Anxiety Inventory (STAI 1) scale revealed that carriers of 21-OHD had significantly higher mean $( \pm \mathrm{SD})$ values of STAI 1 than controls (47.6 \pm 5.6 vs. $43.3 \pm 5.4 ; P=0.023$ ), although they did not differ from their healthy counterparts with respect to trait anxiety scale (STAI 2). Notably, carriers of 21-OHD had higher mean total STAI score values than controls. This finding is of borderline significance but may again indicate an interesting trend (95. \pm 8.4 vs. $91.2 \pm 8.4 ; P=0.087)$. With regard to the occurrence of depression, the comparison of participant responses according to the BDI revealed that carriers of 21-OHD had similar depression scores to those of controls. This finding was sustained by findings arising from the corresponding component scores of the SCL-90R. No differences between the two groups were noted in any of the personality traits evaluated by the TCI (Table 3).

The Pearson correlation analyses revealed that peak 17-OHP concentrations were positively correlated with STAI 1 responses $(\mathrm{r}=0.364 ; P=0.027)$, but not the total STAI score $(\mathrm{r}=0.302 ; P=0.069)$. Furthermore, mean UFC concentrations were significantly correlated with the SCL-90R subscale responses relating to paranoid ideation $(\mathrm{r}=0.435 ; P=0.023)$ and psychoticism $(\mathrm{r}=0.454 ; P=0.017)$, as well as to the TCI subscale of self-transcendence $(\mathrm{r}=0.371 ; P=0.048)$. Finally, stepwise multiple linear regression analyses revealed that among all independent variables assessed, the single independent predictor of state anxiety (as determined by the STAI 1) was peak concentration of 17-OHP ( $\beta$ : 0.055, $\left.\mathrm{R}^{2}: 0.290, P=0.031\right)$.

\section{DISCUSSION}

In this study we demonstrated that carriers of CAH due to 21-OHD had significantly higher peak, mean and AUC 17-OHP concentrations in response to oCRH stimulation and significantly higher state anxiety (STAI 1) scores compared with healthy matched subjects. Moreover, we found that mean 24-hour UFC concentrations were significantly positively correlated with paranoid ideation and psychoticism, while peak 17-OHP concentrations were the only independent predictor of the STAI 1 score. These findings may indicate chronic hyperactivity of the hypothalamic-pituitary axis, which, upon exposure to stressors, might predispose these subjects to the development of affective and/or anxiety disorders.

Table 2. Receiver operator curve analysis for endocrine parameters between carriers of 21-hydroxylase deficiency (21-OHD) and control subjects

\begin{tabular}{lccc}
\hline & Area under the curve (95\% CI) & Standard error (SEM) & $\boldsymbol{P}$ - value \\
\hline ACTH & $0.512(0.301-0.723)$ & 0.108 & 0.905 \\
17-OHP & $0.211(0.059-0.363)$ & 0.078 & $\mathbf{0 . 0 0 3}$ \\
Androstenedione & $0.331(0.157-0.506)$ & 0.089 & 0.089 \\
Cortisol & & & 0.602 \\
Serum cortisol & $0.448(0.252-0.645)$ & 0.100 & 0.117 \\
Salivary cortisol & $0.263(0.045-0.480)$ & 0.111 & \\
\hline
\end{tabular}

95\% CI: 95\% confidence interval 

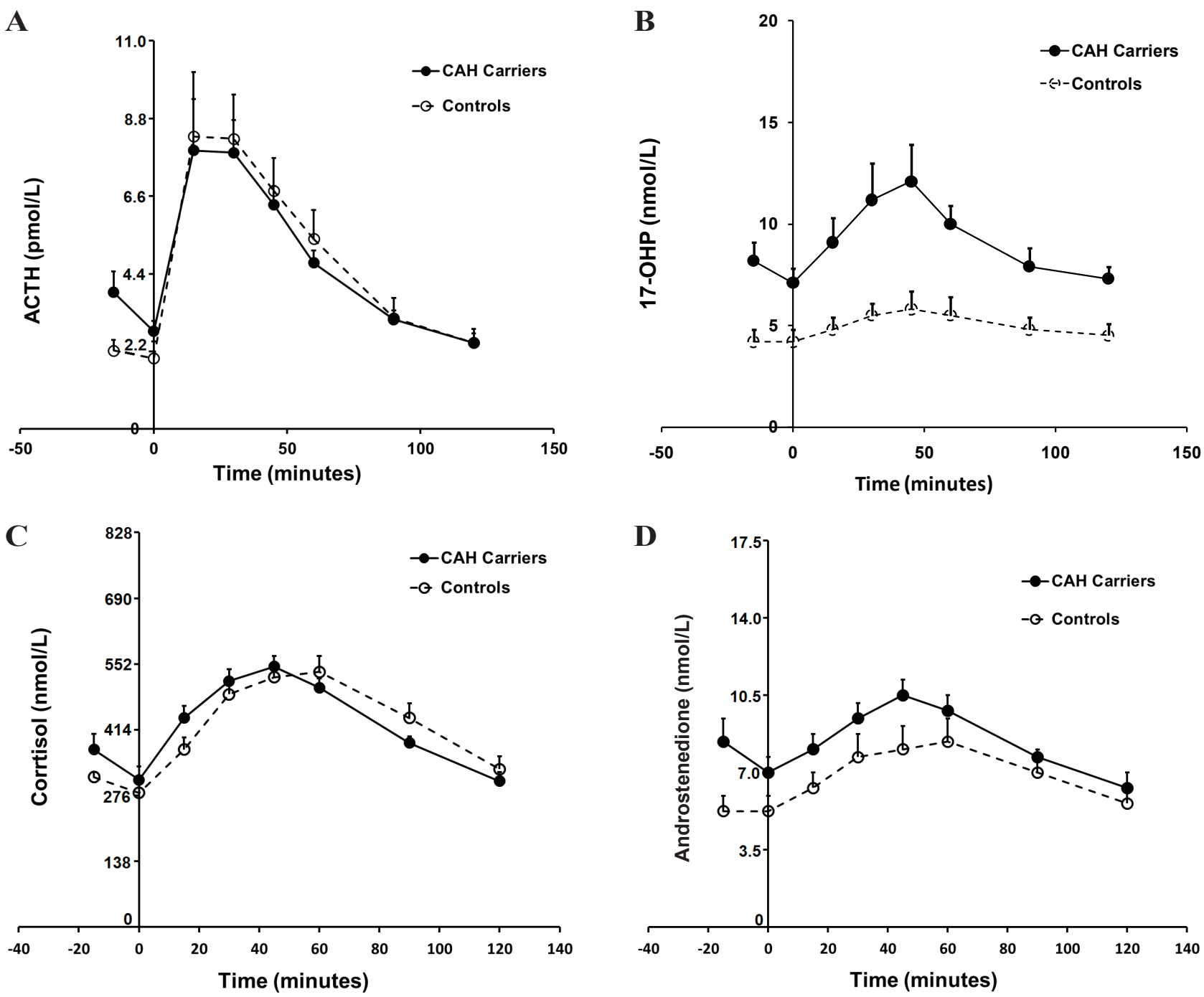

Figure 1. Differential responses of ACTH (Panel A), 17-OHP (Panel B), serum cortisol (Panel C) and androstenedione (Panel D) in carriers of 21-hydroxylase deficiency and control subjects.

Parents of children with either hypothyroidism or Turner syndrome were included in our study as control subjects. Hypothyroidism is the most common endocrine disorder and, if inadequately treated, it may have serious clinical and metabolic complications, including dyslipidemia, neuromuscular dysfunction, cognitive impairment, infertility and an increased risk for cardiovascular disease. On the other hand, Turner syndrome is a genetic disorder with potentially severe associated comorbidities, such as developmental, endocrine, cardiovascular and psychosocial disorders, infertility and poor growth. Thus, parents of children with either hypothyroidism or Turner syndrome were selected as controls on account of the psychological impact secondary to the stress of raising a child with a chronic illness.

The increased peak, mean and AUC 17-OHP concentrations in response to oCRH stimulation in carriers of 21-OHD indicate chronic compensatory hyperresponsiveness of the HPA axis owing to the mild impairment in cortisol biosynthesis. ${ }^{7}$ Moreover, the higher - though not statistically significant - mean $\mathrm{ACTH}$ values at baseline (at -15 and $0 \mathrm{~min}$ ) may point towards the same direction, most likely reflecting a state of mild chronic ACTH hypersecretion.

A large body of clinical and experimental evidence suggests that there could be a critical role of both 
Table 3. Comparison of psychometric parameters between carriers of 21-hydroxylase deficiency (21-OHD) and control subjects

\begin{tabular}{|c|c|c|c|}
\hline & Carriers of 21-OHD (n=29) & Control subjects $(n=13)$ & $P$-value* \\
\hline \multicolumn{4}{|c|}{ State Anxiety Inventory (STAI) } \\
\hline State anxiety (STAI 1) & $47.6 \pm 5.6$ & $43.3 \pm 5.4$ & 0.023 \\
\hline Trait anxiety (STAI 2) & $47.8 \pm 5.2$ & $47.9 \pm 3.7$ & 0.353 \\
\hline Total STAI score & $95.4 \pm 8.4$ & $91.2 \pm 8.4$ & 0.087 \\
\hline \multicolumn{4}{|c|}{ Beck Depression Inventory (BDI) } \\
\hline Depression score & $7.7 \pm 5.7$ & $8.4 \pm 4.0$ & 0.512 \\
\hline \multicolumn{4}{|c|}{ Symptom Checklist - 90R (SCL-90R) } \\
\hline Somatization & $7.3 \pm 7.7$ & $8.0 \pm 5.8$ & 0.395 \\
\hline Obsessive-compulsive & $9.8 \pm 5.7$ & $10.9 \pm 6.0$ & 0.575 \\
\hline Interpersonal sensitivity & $6.4 \pm 4.9$ & $7.0 \pm 2.4$ & 0.151 \\
\hline Depression & $9.8 \pm 8.1$ & $13.8 \pm 7.1$ & 0.071 \\
\hline Anxiety & $6.2 \pm 4.3$ & $6.7 \pm 4.0$ & 0.597 \\
\hline Hostility & $4.5 \pm 4.6$ & $5.6 \pm 4.5$ & 0.432 \\
\hline Phobic anxiety & $2.2 \pm 3.5$ & $1.2 \pm 1.6$ & 0.620 \\
\hline Paranoid ideation & $6.1 \pm 3.8$ & $5.9 \pm 3.4$ & 0.936 \\
\hline Psychoticism & $3.3 \pm 3.2$ & $3.2 \pm 2.6$ & 0.761 \\
\hline Total SCL-90R & $61.1 \pm 38.6$ & $67.8 \pm 27.2$ & 0.327 \\
\hline \multicolumn{4}{|c|}{ Temperament and Character Inventory (TCI) } \\
\hline Novelty seeking & $22.2 \pm 3.1$ & $20.5 \pm 4.2$ & 0.187 \\
\hline Harm avoidance & $16.3 \pm 4.4$ & $13.6 \pm 3.8$ & 0.083 \\
\hline Reward dependence & $13.4 \pm 2.2$ & $13.8 \pm 2.5$ & 0.711 \\
\hline Persistence & $3.2 \pm 1.3$ & $3.5 \pm 1.4$ & 0.690 \\
\hline Self-directedness & $20.5 \pm 5.7$ & $22.0 \pm 5.3$ & 0.345 \\
\hline Cooperativeness & $23.7 \pm 2.9$ & $24.2 \pm 3.6$ & 0.732 \\
\hline Self-transcendence & $15.2 \pm 4.0$ & $15.6 \pm 4.9$ & 0.776 \\
\hline
\end{tabular}

Continuous variables presented as mean $\pm \mathrm{SD} ; *$ Mann-Whitney p-value

I SCL-90R conducted among $\mathrm{n}=37$ (CAH carriers: $\mathrm{n}=27$ vs. Controls: $\mathrm{n}=12$ )

CRH and AVP in the development of affective and anxiety disorders. In rodents, intracerebroventricular administration of CRH increases locomotion, anxiety, fear, despair and emotionality, decreases appetite, sexual activity, exploration and social interaction, and activates the HPA axis and the autonomic nervous system. In addition, single-nucleotide polymorphisms (SNPs) in the genes encoding CRH, CRH-1R, CRH$2 \mathrm{R}$ and $\mathrm{CRH}$-binding protein may be associated with increased susceptibility to psychiatric disorders and may predict the response to antidepressant therapy. ${ }^{8,24,25}$ Preclinical and clinical evidence thus points to the therapeutic potential for CRH type 1 receptor antagonists, such as antalarmin, in the treatment of anxiety and depression. ${ }^{26-30}$ Furthermore, AVP has been related to enforced stress-reaction, trait anxiety and depression. ${ }^{27,31}$

Anxiety disorders are a group of psychiatric disorders that may significantly impair the quality of life of affected individuals. Those include GAD, PD, phobic disorders [i.e. specific phobias, agoraphobia, social phobia (SP)], post-traumatic stress disorder (PTSD) and OCD. Several abnormalities in the HPA axis function in patients with anxiety disorders have been reported, however, results are conflicting. In patients with PD, 
both normal baseline activity and hyperresponsiveness of the HPA axis have been described. ${ }^{13,32} \mathrm{SP}$ is also associated with hyperresponsiveness of the HPA axis after exposure to psychosocial stressors. ${ }^{32}$ On the other hand, patients with PTSD typically display a hypoactive HPA axis, although they have higher cerebrospinal fluid (CSF) CRH concentrations and smaller hippocampal volume than healthy controls. ${ }^{13,32}$ The neuroendocrine alterations in PTSD have been attributed to a developmental programming secondary to early-life trauma. The latter may result in tissuespecific alterations in glucocorticoid sensitivity, enhanced negative feedback inhibition of the HPA axis, changes in glucocorticoid metabolism or low cortisol concentrations through epigenetic mechanisms. ${ }^{33}$ Increased concentrations of CRH and AVP in plasma and CSF have been reported in patients with OCD, suggesting a hyperactive state of the HPA axis. ${ }^{32}$ In GAD, data on HPA axis activity are inconsistent, ${ }^{32,34}$ owing partly to the use of different diagnostic tools employed for the assessment of HPA axis function. Overall, it has been postulated that the persistent HPA axis hyperactivity observed in GAD may be mediated by changes in the sensitivity or in the number of CRH and/or glucocorticoid receptors in brain areas associated with anxiety disorders, including the amygdala, hippocampus and the prefrontal cortex.

Our findings of significantly higher STAI 1 scores in carriers of 21-OHD than the control subjects concur with the above data and may reflect an association between chronic CRH hypersecretion, due to HPA axis compensatory hyperactivity, and increased anxiety in these subjects. The significant positive correlation between peak 17-OHP concentrations and STAI 1 responses and the fact that peak 17-OHP concentration was an independent predictor of state anxiety point towards the same direction, possibly linking the degree of cortisol synthesis impairment to the severity of anxiety-related symptoms. Furthermore, the findings of higher total STAI scores in carriers of 21-OHD, although not statistically significant, may again indicate an interesting trend of carriers towards states of anxiety.

Stress in early prenatal and postnatal life, such as parental separation during early childhood, childhood abuse and neglect or childhood parental loss, has been associated with long-lasting neuroendocrine altera- tions in HPA axis function. Similarly, a persistent sensitization of the HPA axis in carriers of 21-OHD, which is already present during the prenatal life, and a subsequently reduced glucocorticoid receptormediated negative feedback on the HPA axis, may lead to permanent alterations in HPA axis regulation, predisposing these subjects to stress-related disorders in adult life. Furthermore, an increased interaction between chronically elevated $\mathrm{CRH}$ and its type 1 receptor, altered receptor sensitivity or enhanced signaling capacity may result in higher state anxiety, possibly through transcriptional and/or epigenetic mechanisms. ${ }^{14,32}$ In contrast, after prolonged periods of chronic stress an initially hyperactive HPA axis eventually evolves into a hypoactive state through down-regulation of CRH receptors in the pituitary, resulting in lower ACTH and cortisol secretion. ${ }^{35,36}$ Hypoactivity of the HPA axis has been described in several physical and psychiatric disorders, including atypical depression, PTSD, chronic fatigue syndrome and fibromyalgia..$^{9,12,37}$

In our previous study, carriers of 21-OHD were shown to display mild hypocortisolism, as indicated by lower 24-hour UFC excretion compared with controls. Moreover, their 24-hour UFC concentrations correlated negatively with obsessive-compulsive behavior, novelty seeking and reward dependence. ${ }^{7}$ Although a similar finding was not shown in the present study, a relative hypocortisolemic state of carriers of 21-OHD owing to their enzymatic defect can be postulated. This mild hypocortisolism might contribute to the decreased ability of these subjects to cope adequately with stress, both physically and emotionally. It is important to note that both hypocortisolism and hypercortisolism may be associated with different types of personality traits and/or psychopathology. ${ }^{12,38}$ Homeostatic mechanisms, including the stress system, exert their effects in an inverted U-shaped dose-response curve (Figure 2). Basal, healthy homeostasis or eustasis is achieved in the central, optimal range of the curve, whereas suboptimal effects may occur on either side of the curve and can lead to insufficient adaptation, a state that has been called allostasis or cacostasis. The latter states of hypofunction or hyperfunction of the stress system may have short-term or longterm adverse consequences for the individual and 


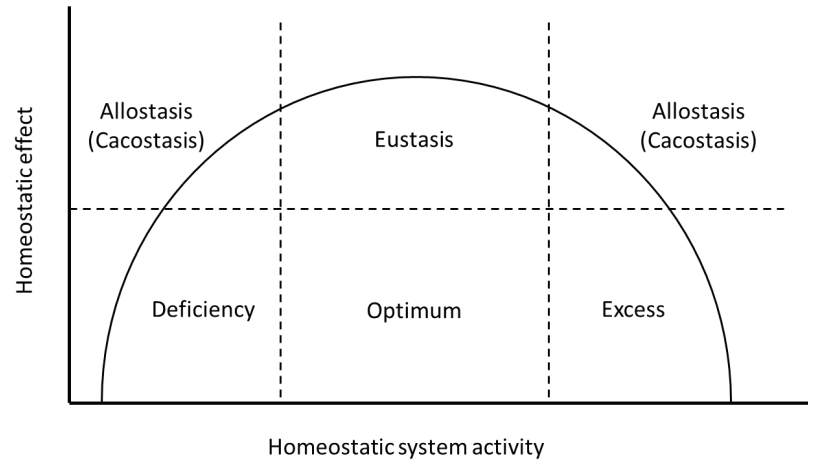

Figure 2. Schematic representation of the interrelation between $\mathrm{CRH}$ or cortisol concentrations and sense of well-being or performance. Normal CRH/cortisol secretion is associated with optimum sense of well-being, whereas both $\mathrm{CRH}$ and cortisol hyposecretion or hypersecretion lead to compromised sense of well-being and/or performance. Whereas most normal subjects fall within the optimum range, we hypothesize that carriers of 21-hydroxylase deficiency have shifts toward the right or left of the optimum range because of high $\mathrm{CRH}$ and low cortisol concentrations, respectively. These shifts may explain the correlation between altered HPA axis activity and certain psychological parameters.

may lead to compromised sense of well-being and/ or performance. ${ }^{8}$ Accordingly, we hypothesize that carriers of 21-OHD have shifts toward the right or left of the optimum range because of high $\mathrm{CRH}$ and low cortisol concentrations, respectively, which may explain the correlations between altered HPA axis activity and certain psychological parameters. Finally, we observed a positive correlation between 24-hour UFC concentrations with paranoid ideation and psychoticism. These results are in accordance with previous studies showing that among patients with major depression, psychotic depression was associated with significantly higher rates of non-suppression of cortisol in the dexamethasone-suppression test. ${ }^{39}$

The main strength of our study is the administration of four different psychometric instruments for the assessment of anxiety and depression-related symptoms. Moreover, cortisol concentrations were measured in different samples, including 24-hour urine collections, serum and multiple saliva specimens, to avoid variations due to acute stress responses. However, several limitations of this study should be acknowledged. The number of participants is relatively small; therefore, larger studies are required to elucidate further the association between psychological factors and endocrinologic parameters in carriers of 21-OHD. Furthermore, the adverse socioeconomic environment and the subsequent increased stress imposed on many families that participated in the study could be possible confounding factors, influencing the cortisol responses to oCRH stimulation and anxiety/depression scores in our study participants.

We conclude that carriers of 21-hydroxylase deficiency have increased 17-OHP responses to oCRH stimulation and significantly higher state anxiety scores compared with normal controls. In addition, the severity of anxiety-related symptoms correlated positively with peak 17-OHP concentrations. The above findings indicate a decreased negative feedback inhibition at the hypothalamic and pituitary levels, which may lead to chronic hyperactivity of the HPA axis and, upon further exposure to adverse environmental factors, might predispose these subjects to the development of physical, affective and/ or anxiety disorders.

\section{REFERENCES}

1. Merke DP, Bornstein SR, 2005 Congenital adrenal hyperplasia. Lancet, 365: 2125-2136.

2. Speiser PW, White PC, 2003 Congenital adrenal hyperplasia. N Engl J Med 349: 776-788.

3. Merke DP, Poppas DP, 2013 Management of adolescents with congenital adrenal hyperplasia. Lancet Diabetes Endocrinol 1: 341-352.

4. Nimkarn S, Lin-Su K, New MI, 2011 Steroid 21 hydroxylase deficiency congenital adrenal hyperplasia. Pediatr Clin North Am 58: 1281-1300, xii.

5. Baumgartner-Parzer SM, Nowotny P, Heinze G, Waldhäusl W, Vierhapper H, 2005 Carrier frequency of congenital adrenal hyperplasia (21-hydroxylase deficiency) in a middle European population. J Clin Endocrinol Metab 90: 775-778.

6. Napolitano E, Manieri C, Restivo F, et al, 2011 Correlation between genotype and hormonal levels in heterozygous mutation carriers and non-carriers of 21-hydroxylase deficiency. J Endocrinol Invest 34: 498-501.

7. Charmandari E, Merke DP, Negro PJ, et al, 2004 Endocrinologic and psychologic evaluation of 21-hydroxylase deficiency carriers and matched normal subjects: evidence for physical and/or psychologic vulnerability to stress. J Clin Endocrinol Metab 89: 2228-2236.

8. Chrousos GP, 2009 Stress and disorders of the stress system. Nat Rev Endocrinol 5: 374-381.

9. Charmandari E, Tsigos C, Chrousos G, 2005 Endocrinology of the stress response. Annu Rev Physiol 67: 259-284. 
10. Nicolaides NC, Kyratzi E, Lamprokostopoulou A, Chrousos GP, Charmandari E-M, 2015 Stress, the stress system and the role of glucocorticoids. Neuroimmunomodulation 22: 6-19.

11. Chrousos GP, 1995 The hypothalamic-pituitary-adrenal axis and immune-mediated inflammation. N Engl J Med 332: 1351-1362.

12. Gold PW, Chrousos GP, 2002 Organization of the stress system and its dysregulation in melancholic and atypical depression: high vs. low CRH/NE states. Mol Psychiatry 7: 254-275.

13. Risbrough VB, Stein MB, 2006 Role of corticotropin releasing factor in anxiety disorders: a translational research perspective. Horm Behav 50: 550-561.

14. Charmandari E, Kino T, Souvatzoglou E, Chrousos GP, 2003 Pediatric stress: hormonal mediators and human development. Horm Res 59: 161-179.

15. Kunugi H, Ida I, Owashi, T, et al, 2006 Assessment of the dexamethasone/CRH test as a state-dependent marker for hypothalamic-pituitary-adrenal (HPA) axis abnormalities in major depressive episode: a Multicenter Study. Neuropsychopharmacology 31: 212-220.

16. Spielberger CD, 1983 Manual for the State-Trait Anxiety Inventory (Form Y). Palo Alto, CA: Consulting Psychologists Press.

17. Beck AT, Beamesderfer A, 1974 Assessment of depression: the depression inventory. In: Pichot P (ed) Psychological measurements in psychopharmacology. Modern problems in pharmacopsychiatry, Vol 7: Basel, Switzerland: Karger; pp, 151-169.

18. Derogatis LR, 1983 SCL-90-R: administration, scoring and procedures manual II. Towson, MD: Clinical Psychometric Research.

19. Cloninger CR, Przybeck TR, Svrakic DM, 1991 The Tridimensional Personality Questionnaire: U.S. normative data. Psychol Rep 69: 1047-1057.

20. Fountoulakis KN, Papadopoulou M, Kleanthous S, et al, 2006 Reliability and psychometric properties of the Greek translation of the State-Trait Anxiety Inventory form Y: preliminary data. Ann Gen Psychiatry 5: 2.

21. Mystakidou K, Tsilika E, Parpa E, Smyrniotis V, Galanos A, Vlahos L, 2007 Beck Depression Inventory: exploring its psychometric properties in a palliative care population of advanced cancer patients. Eur J Cancer Care 16: 244-250.

22. Donias S, Karastergiou A, Manos N, 1991 Standardization of the symptom checklist-90-R rating scale in a Greek population. Psychiatriki 2: 42-48.

23. Fountoulakis KN, Rozsa S, Siamouli M, Moutou K, Pantoula E, Cloninger CR, 2015 Standardization and normative data of the Greek version of the temperament and character inventory (TCI). Ann Gen Psychiatry 14: 28.

24. Laryea G, Arnett MG, Muglia LJ, 2012 Behavioral Studies and Genetic Alterations in Corticotropin-Releasing Hormone (CRH) Neurocircuitry: Insights into Human Psychiatric Disorders. Behav Sci (Basel, Switzerland)
2: $135-171$.

25. Gold PW, Chrousos GP, 2013 Melancholic and atypical subtypes of depression represent distinct pathophysiological entities: CRH, neural circuits, and the diathesis for anxiety and depression. Mol Psychiatry 18: 632-634.

26. Ising M, Holsboer F, 2007 CRH-sub-1 receptor antagonists for the treatment of depression and anxiety. Exp Clin Psychopharmacol 15: 519-528.

27. Holsboer F, Ising M, 2008 Central CRH system in depression and anxiety--evidence from clinical studies with CRH1 receptor antagonists. Eur J Pharmacol 583: 350-357.

28. Habib KE, Weld KP, Rice KC, et al, 2000 Oral administration of a corticotropin-releasing hormone receptor antagonist significantly attenuates behavioral, neuroendocrine, and autonomic responses to stress in primates. Proc Nati Acad Sci USA 97: 6079-6084.

29. Ayala AR, Pushkas J, Higley JD, et al, 2004 Behavioral, adrenal, and sympathetic responses to long-term administration of an oral corticotropin-releasing hormone receptor antagonist in a primate stress paradigm. J Clin Endocrinol Metabol 89: 5729-5737.

30. Zoumakis E, Rice KC, Gold PW, Chrousos GP, 2006 Potential uses of corticotropin-releasing hormone antagonists. Ann NY Acad Sci 1083: 239-251.

31. Bao AM, Meynen G, Swaab DF, 2008 The stress system in depression and neurodegeneration: focus on the human hypothalamus. Brain Res Rev 57: 531-553.

32. Faravelli C, Lo Sauro C, Godini L, et al, 2012 Childhood stressful events, HPA axis and anxiety disorders. World J Psychiatry 2: 13-25.

33. Yehuda R, Seckl J, 2011 Minireview: Stress-related psychiatric disorders with low cortisol levels: a metabolic hypothesis. Endocrinology 152: 4496-4503.

34. Mantella RC, Butters MA, Amico JA, et al, 2008 Salivary cortisol is associated with diagnosis and severity of late-life generalized anxiety disorder. Psychoneuroendocrinology 33: 773-781.

35. Penninx BW, Beekman AT, Bandinelli S, et al, 2007 Late-life depressive symptoms are associated with both hyperactivity and hypoactivity of the hypothalamopituitary-adrenal axis. Am J Geriatr Psychiatry 15: 522-529.

36. Vreeburg SA, Hoogendijk WJ, DeRijk RH, et al, 2013 Salivary cortisol levels and the 2-year course of depressive and anxiety disorders. Psychoneuroendocrinology 38: 1494-1502.

37. Tsigos C, Chrousos GP, 2002 Hypothalamic-pituitaryadrenal axis, neuroendocrine factors and stress. J Psychosom Res 53: 865-871.

38. Hori H, Ozeki Y, Teraishi T, et al, 2010 Relationships between psychological distress, coping styles, and HPA axis reactivity in healthy adults. J Psychiatr Res 44: 865-873.

39. Nelson JC, Davis JM, 1997 DST studies in psychotic depression: a meta-analysis. Am J Psychiatry 154: 1497-1503. 